\title{
Utility of a Y-configured stentriever technique as a rescue method of thrombectomy for an intractable rooted thrombus located on the middle cerebral artery bifurcation: technical note
}

\author{
Hideo Okada, MD,, 1,2 Yoshikazu Matsuda, MD, ${ }^{1,5}$ Joonho Chung, MD, PhD, ,3,4 \\ R. Webster Crowley, MD, ${ }^{1}$ and Demetrius K. Lopes, MD'
}

'Department of Neurological Surgery, Rush University Medical Center, Chicago, Illinois; ${ }^{2}$ Department of Neurosurgery, Wakayama Rosai Hospital, Wakayama City, Japan; ${ }^{2}$ Department of Neurosurgery, Gangnam Severance Hospital, and ${ }^{4}$ Severance Institute for Vascular and Metabolic Research, Yonsei University College of Medicine, Seoul, Republic of Korea; and ${ }^{5}$ Department of Neurosurgery, Wakayama Medical University, Wakayama City, Japan

Mechanical thrombectomy with stentriever and/or aspiration is the new gold standard for the treatment of acute strokes with large-vessel occlusion. As many as $20 \%$ of cases remain refractory to current stentriever and/or aspiration devices. "Saddle clots" obstructing a bifurcation may be a particular challenge for recanalization with conventional techniques and devices. The authors describe an alternative technique to bifurcation occlusions resistant to the conventional mechanical thrombectomy approach in which they simultaneously deployed 2 stentrievers into both branches of an occluded bifurcation. This stentriever Y-configuration was very effective in managing a challenging intracranial bifurcation occlusion.

https://thejns.org/doi/abs/10.3171/2017.1.FOCUS16511

KEY WORDS emergency large-vessel occlusion; mechanical thrombectomy; stentriever; penumbra; rooted thrombus

$\mathrm{R}$ ECENT randomized controlled trials have shown that acute stroke outcomes are improved by mechanical thrombectomy (MT) $)^{3,4,7,8,13}$ Standardization of the MT technique in patients with emergency large-vessel occlusion improved recanalization rates to Thrombolysis in Cerebral Infarction (TICI) Grades $2 \mathrm{~b}$ and 3 from 58\%$72 \%$ in early experience to $79 \%-88 \%$ more recently. $3,10,15$ The most widely used MT techniques involve stentriever and/or aspiration. Some conjunctive methods, such as stentriever and a balloon guide catheter or stentriever and a large-bore aspiration catheter (stentriever-aspiration, or Solumbra, technique), have been reported to improve the recanalization rate; ${ }^{69}$ however, debate exists regarding how to achieve optimal recanalization safely and effectively in refractory cases. In this report, we describe the Y-configured stentriever technique combining 2 Trevo ProVue devices (Stryker Neurovascular) with an ACE64 catheter (Penumbra) for a strongly rooted thrombus on the middle cerebral artery (MCA) bifurcation, focusing on its feasibility, technical advantages and disadvantages, and its strategic positioning.

\section{Case Report \\ History and Examination}

An 80-year-old man with medical history of hypertension, atrial fibrillation on rivaroxaban, and benign prostatic hypertrophy developed right hemiparesis and aphasia. The patient had discontinued his rivaroxaban for transurethral surgery 2 days before his stroke. He was not a candidate for intravenous tissue plasminogen activator (tPA) due to his recent surgery, and he was transferred to our hospital for consideration of mechanical thrombectomy. Once at our institution, CT angiography showed complete occlusion of the left MCA.

\section{Endovascular Procedure}

An 8-Fr intravascular sheath was inserted into the right common femoral artery under local anesthesia. An 8-Fr Flowgate balloon guide catheter (Stryker Neurovascular) over a 5-Fr VTK catheter was positioned in the left common carotid artery, and initial diagnostic angiography confirmed complete occlusion of the left MCA. Although

ABBREVIATIONS ICA = internal carotid artery; MCA = middle cerebral artery; $\mathrm{MT}=$ mechanical thrombectomy; $\mathrm{TICI}=\mathrm{Thrombolysis}$ in Cerebral Infarction; tPA = tissue plasminogen activator.

SUBMITTED December 1, 2016. ACCEPTED January 6, 2017.

INCLUDE WHEN CITING DOI: 10.3171/2017.1.FOCUS16511. 


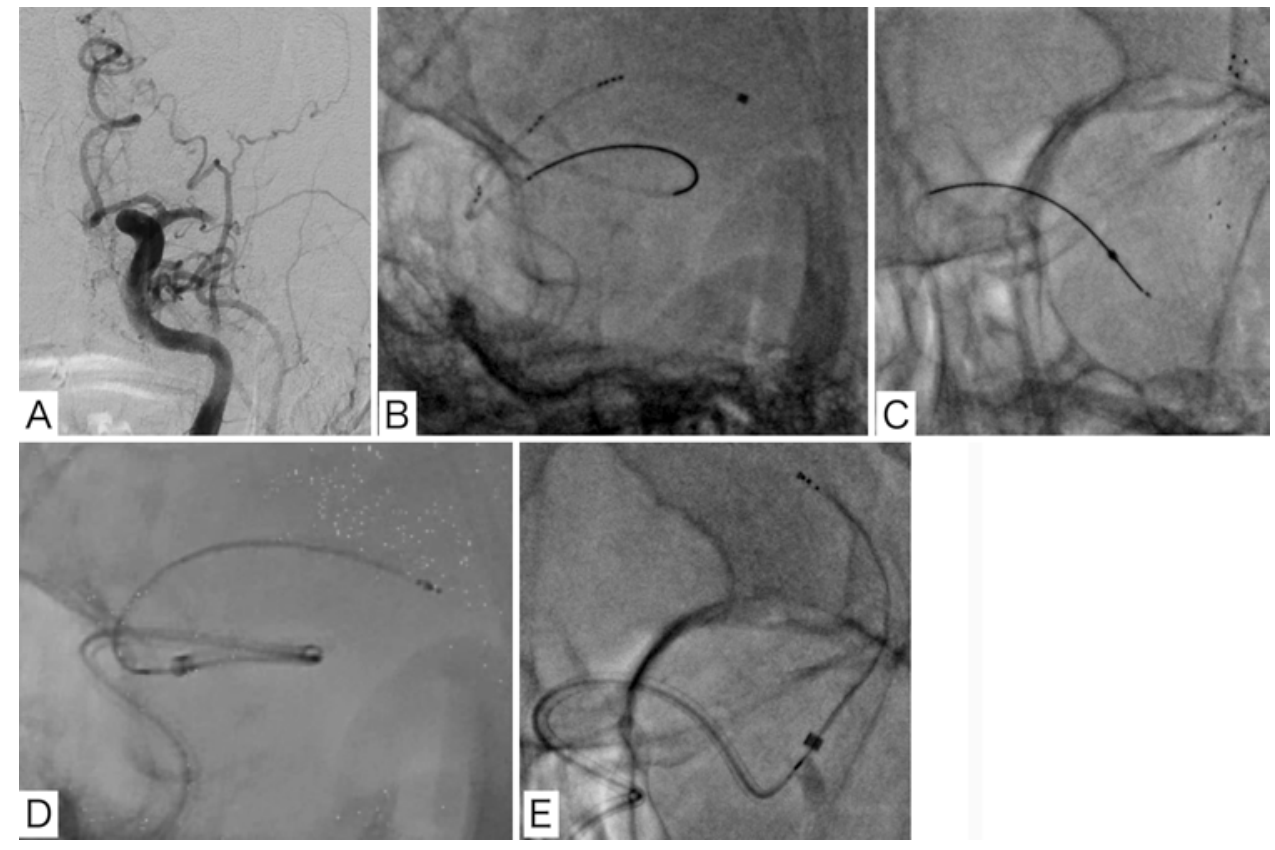

FIG. 1. Pretreatment angiogram and failed thrombectomy procedure by single stentriever and aspiration technique. A: Angiogram (anterior projection) obtained before the procedure, showing total occlusion of the left MCA. B: Fluoroscopic image (lateral projection) showing the microcatheter advancing into one branch of the inferior division of the left MCA before deployment. C: Fluoroscopic image (anterior projection) after deployment of the Solitaire Platinum device as a first pass, showing full expansion of all radiopaque markers. D: Fluoroscopic image (lateral projection) obtained just before the Trevo ProVue deployment as the second thrombectomy attempt. Note that the devices are navigated in the same branch. E: Fluoroscopic image (anterior projection) obtained just before the Trevo ProVue deployment as the second thrombectomy attempt. Note that the ACE64 advanced into the $\mathrm{M}_{2}$ segment.

we would have preferred to advance the Flowgate catheter into the internal carotid artery (ICA), extreme tortuosity of the ICA limited our ability to advance the catheter further distally. For our initial thrombectomy attempt, we used the stentriever-aspiration technique. A triaxial system with a large-bore aspiration catheter (ACE64, Penumbra) over a 0.027-inch microcatheter (Marksman, Covidien) on top of a 0.014-inch microwire (Synchro, Stryker Neurovascular) was introduced into the balloon guide catheter. The balloon of the guide catheter was inflated, and the microwire and the microcatheter were advanced without difficulty into one main inferior branch of the MCA. A $4 \times 40-\mathrm{mm}$ Solitaire Revascularization Device (ev3/Medtronic) was deployed across the thrombus with complete opening of each marker, and the Marksman catheter was removed from the patient. Continuous aspiration was applied to the ACE64 via the aspiration tubing, and the catheter was advanced to the interface of the clot, signified by decreased return of blood through the aspiration tubing. Once there, the Solitaire was dragged partially back into the ACE64, followed by careful removal of the Solitaire and the ACE64 as a single unit. Continuous aspiration using the Penumbra pump applied to the ACE64 and manual aspiration with a 60-ml syringe applied to the balloon guide catheter were maintained during withdrawal of the Solitaire device and the ACE64. A subsequent angiogram revealed failure of recanalization by this first attempt. A second pass was performed using the same technique, this time using a 4 $\times 30-\mathrm{mm}$ Trevo ProVue (Stryker Neurovascular) through the associated 0.021-inch microcatheter (Trevo Pro 18,
Stryker Neurovascular). This was again advanced into the same $\mathrm{M}_{2}$ branch, with distal advancement of the ACE64; however, recanalization again was not achieved (Fig. 1).

\section{Y-Stent Retriever Method}

After 2 failed attempts using the standard stentrieveraspiration catheter technique, it was felt that the recalcitrance might be related to the clot's involvement in both branches of the MCA bifurcation. We therefore felt that it might be advantageous to catheterize both of these branches, creating a Y-stent configuration, in an attempt to better capture the thromboembolus. The Flowgate device was kept in place, and the ACE64 over the Trevo microcatheter was again advanced into the same $\mathrm{M}_{2}$ branch that had been previously catheterized. The $4 \times 30-\mathrm{mm}$ Trevo ProVue was deployed across the thrombus from the $\mathrm{M}_{2}$ segment into the $\mathrm{M}_{1}$ segment, and the microcatheter was removed from the patient. The microcatheter was then reintroduced over the Synchro microwire within the ACE64, in a parallel fashion to the wire of the already deployed Trevo ProVue. With careful manipulation, the wire and microcatheter were successfully navigated past the in situ Trevo, into the second MCA branch. A $4 \times 20-\mathrm{mm}$ Trevo ProVue device was then deployed across the thrombus, creating a Y-configuration stentriever construct with both devices covering the origins of the $\mathrm{M}_{2}$ branches at the bifurcation. Tension was then applied to both stentriever wires, and the ACE64 with applied aspiration was advanced forward over the wires until a portion of the Trevo devices was inside the ACE64. The Trevo devices and ACE64 were then care- 
fully removed from the patient's body under continuous aspiration of the ACE64 and Flowgate. This resulted in the removal of a substantial amount of thrombus, with recanalization of the inferior trunk (Fig. 2).

Additional thrombectomy was then performed using the standard stentriever-aspiration technique to remove the residual thrombus in the superior trunk of the MCA, with TICI Grade 2b recanalization (Fig. 3). A head CT scan obtained the next day showed no intracranial hemorrhage.

\section{Discussion}

Here, we describe a novel rescue technique of mechani- cal thrombectomy in detail, combining 2 stentrievers in a Y-configuration and a large-bore aspiration through a balloon guide catheter. A similar method was introduced in a previous case report using a device that is unavailable in the United States, ${ }^{2}$ however, the technical aspects including its potential advantages and disadvantages in practical use have not yet been profoundly discussed. This is the first report to evaluate this technique in detail including its theoretical benefit and strategic positioning.

Recent development of interventional techniques have achieved high recanalization rates. However, standard techniques are not able to easily or adequately capture a fair number of recalcitrant thrombi. Successful revascularization rates range between $58 \%$ and $83 \%$ with stan-
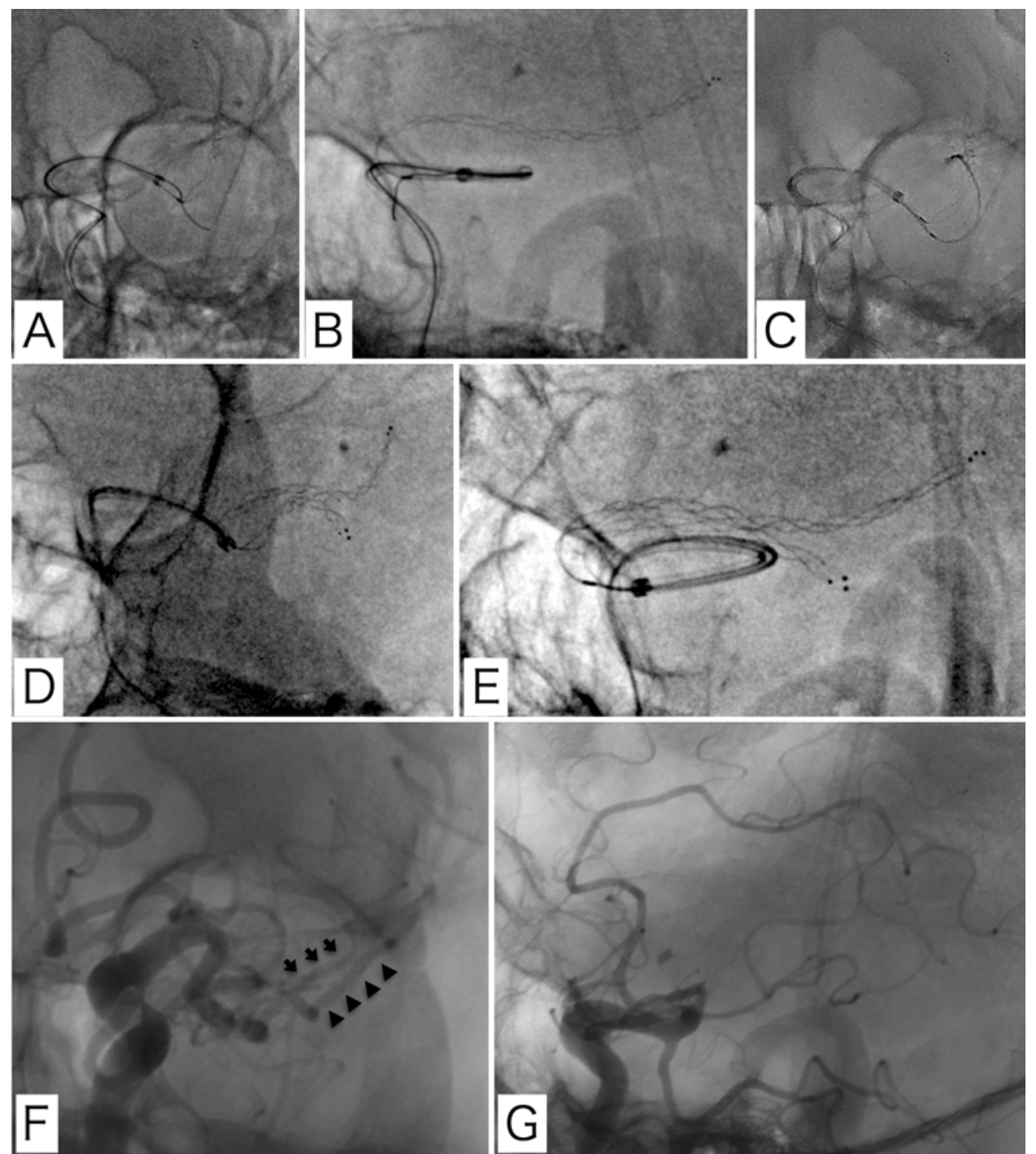

FIG. 2. Images of the Y-stentriever technique using double Trevo ProVue devices as the third attempt. A and B: Fluoroscopic images (anterior $[\mathrm{A}]$ and lateral $[\mathrm{B}]$ projections) after the first Trevo ProVue $(4 \times 30 \mathrm{~mm})$ deployment. The stentriever is in the same branch as the first and second thrombectomy procedures. The microwire is advancing into another branch of the inferior division. C: Fluoroscopic image (anterior projection) obtained just before the second Trevo ProVue $(4 \times 20 \mathrm{~cm})$ deployment. D and E: Fluoroscopic images (left anterior-oblique [D] and lateral [E] projections) obtained after deployment of both stentrievers. The entire length of both stentrievers is well identifiable. F: Angiogram (anterior projection) obtained just after the third thrombectomy using the Y-stentriever technique, showing recanalization of the $2 \mathrm{M}_{2}$ branches from the inferior division (arrows and arrowheads). G: Angiogram (lateral projection) obtained just after the third thrombectomy using the Y-stentriever technique, showing partial recanalization of the inferior division. 

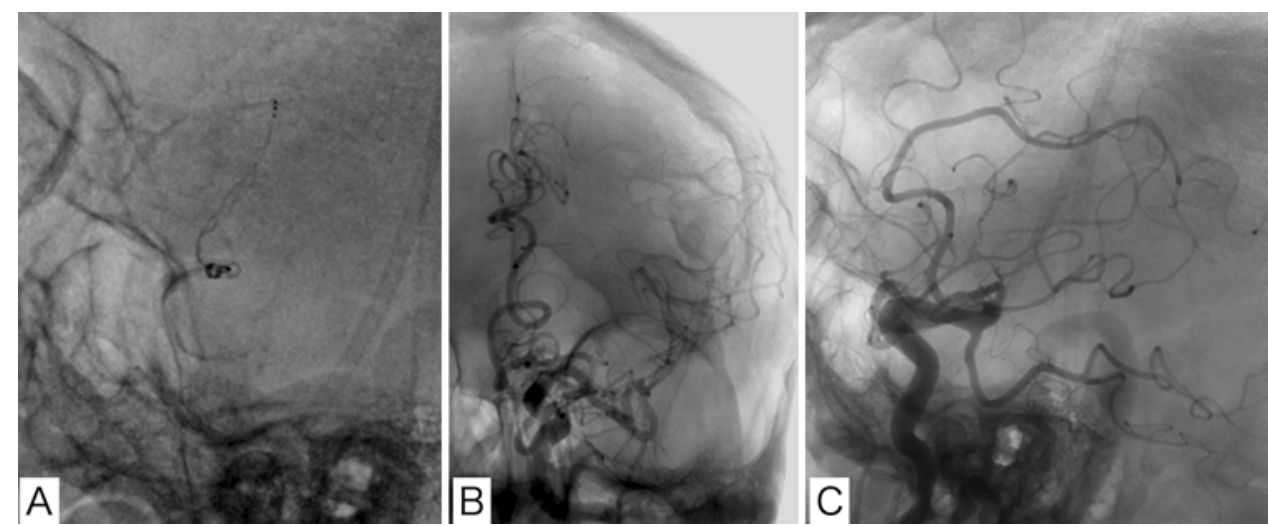

FIG. 3. Additional thrombectomy for the superior division of $\mathrm{M}_{2}$ and final angiogram. A: Lateral projection after the last thrombectomy procedure. The Trevo ProVue is deployed in the superior division of $\mathrm{M}_{2}$. B and C: Final angiogram (anterior $[\mathrm{B}]$ and lateral [C] projections). $\mathrm{TICI}$ Grade $2 \mathrm{~b}$ recanalization is achieved.

dard stentriever techniques ${ }^{3,7,15}$ and $79 \%-83 \%$ using a direct aspiration first-pass thrombectomy technique with a large-bore aspiration catheter. ${ }^{5,15}$ Conjunctive use of stentriever and a large-bore aspiration catheter (stent retriever and aspiration technique) has been introduced ${ }^{6,9}$ and was reported to achieve an $84 \%-88 \%$ recanalization rate in recent case series. More than $80 \%$ of patients could be successfully treated by these well-reported techniques, but it suggests that at least 10\%-20\% of emergency large-vessel occlusion cases are difficult to treat successfully.

Vascular tortuosity, high clot burden, and, particularly, hard or adherent clots are thought to be potential risk factors that decrease the possibility of successful recanalization from mechanical thrombectomy. Recent advancements in MT technology and rapidly accumulating experience with these techniques have allowed us to overcome many of these problems. Improving navigability of stentrievers and aspiration catheters has made distal access achievable, even in tortuous cerebral vasculature. The advancement of aspiration techniques through improved large-bore catheters and balloon guide catheters has given us more opportunity to recanalize large clot burden successfully and more effectively with less showering of distal emboli. These improved catheters have also made retrieving hard clots or adherent clots more attainable, even if the clot is not fully involved inside the stent. ${ }^{14}$ While conjunctive use of a stentriever and aspiration technique possibly compensate for disadvantages with each device, difficulty remains in cases with multiple risk factors.

Although MCA occlusion is considered to be a good option for successful thrombectomy, there are some intractable cases. In many of these cases, a large thrombus may extend from the $\mathrm{M}_{1}$ trunk to multiple $\mathrm{M}_{2}$ branches as if it were rooting. In these cases, thrombectomy involving a single vessel may not be sufficient to achieve successful recanalization; therefore, access to each $\mathrm{M}_{2}$ branch may be necessary for successful recanalization. This may be difficult to achieve, however, as accessibility could be potentially limited by anatomical conditions, such as a high degree of angulation between $\mathrm{M}_{1}$ and $\mathrm{M}_{2}$, atherosclerotic changes with stenosis of the MCA bifurcation, or a hard and adherent thrombus obstructing navigation of a microwire.
The Y-configuration stentriever technique has several advantages. First, deploying the first stent in the most accessible branch may facilitate catheterization of the more difficult branch by deflecting the microcatheter or microwire toward the second branch. In addition, although this did not occur in our case, this technique may allow for the retrieval of large and adherent thrombi rooted into multiple branches as a single piece rather than in a piecemeal fashion, which may decrease showering of distal emboli. Lastly, it can potentially shorten the procedure time while increasing the recanalization rate in some refractory cases.

Clearly, access to each involved branch is not necessary in most cases. In actuality, the majority of saddle clots are retrievable without a Y-stentriever technique. A stentriever deployed from an occluded $\mathrm{M}_{2}$ into a patent $\mathrm{M}_{1}$ trunk often recanalizes additional occluded $\mathbf{M}_{2}$ branches by capturing only the proximal side of the clot and dragging it out without direct access ("tip of the iceberg" technique). ${ }^{1}$ In addition, some clots are one large adherent mass, and capturing half of it within a stentriever may be sufficient to ensure that the entire piece is removed. Therefore, a standard technique should be attempted as the first-line procedure in every case of saddle embolus. In the present case, a Y-stentriever technique was used after 2 failed attempts with the conventional technique. If it were possible to predict when to use this technique, it would obviously decrease procedural times; however, it is difficult to determine when this advanced technique should be applied. Therefore, the endovascular surgeon should keep this technique in mind as a rescue method for refractory cases, should the case arise.

Of course, it is important to recognize the potential disadvantages of this technique. Pulling out 2 stentrievers simultaneously may overload the target vessel. Intimal injury or vessel perforation has been reported in the use of stentrievers, ${ }^{11,12}$ and it is unclear how the removal of 2 stentrievers simultaneously would alter this. If strong resistance is met, it may be necessary to pull out each stentriever individually to avoid vessel injury. In addition, this procedure is rather complicated, and therefore only recommended as a rescue technique for intractable cases, as standard techniques achieve favorable outcome in $80 \%$ of cases. Lastly, it is important to remain cognizant of how 
the stentrievers interact with each other to avoid complex entanglements that could prohibit removal of the devices. Trevo ProVue stentrievers may have an advantage for this technique over other stentrievers, due to their visibility and closed-cell stent structure. Its closed-cell structure may help avoid entanglement while retrieving the devices, and the visibility enables the surgeon to know exactly how they are interacting. This is, however, more of a theoretical concern, and other existing stentrievers may be equally as effective and safe.

\section{Conclusions}

We present a case of mechanical thrombectomy using a Y-stent configuration with 2 Trevo ProVue stentrievers for intractable MCA occlusion that was refractory to standard endovascular techniques. This method may be advantageous not only for retrieving an adherent clot rooted across the MCA bifurcation, but also for accessing highly angulated MCA branches. In practical use, it may be a reasonable salvage technique for intractable cases after standard stentriever, direct aspiration first-pass thrombectomy, or single stentriever and aspiration techniques have failed. The risks and benefits in clinical use should be assessed in a large series.

\section{References}

1. Agid R, Power S: Alternative technique for clot retrieval: the "tip of the iceberg" technique. Interv Neuroradiol 21:703706, 2015

2. Asadi H, Brennan P, Martin A, Looby S, O'Hare A, Thornton J: Double stent-retriever technique in endovascular treatment of middle cerebral artery saddle embolus. J Stroke Cerebrovasc Dis 25:e9-e11, 2016

3. Berkhemer OA, Fransen PS, Beumer D, van den Berg LA, Lingsma HF, Yoo AJ, et al: A randomized trial of intraarterial treatment for acute ischemic stroke. N Engl J Med 372:11-20, 2015

4. Campbell BC, Mitchell PJ, Kleinig TJ, Dewey HM, Churilov L, Yassi N, et al: Endovascular therapy for ischemic stroke with perfusion-imaging selection. N Engl J Med 372:10091018, 2015

5. Delgado Almandoz JE, Kayan Y, Young ML, Fease JL, Scholz JM, Milner AM, et al: Comparison of clinical outcomes in patients with acute ischemic strokes treated with mechanical thrombectomy using either Solumbra or ADAPT techniques. J Neurointerv Surg 8:1123-1128, 2016

6. Deshaies EM: Tri-axial system using the Solitaire-FR and Penumbra Aspiration Microcatheter for acute mechanical thrombectomy. J Clin Neurosci 20:1303-1305, 2013

7. Goyal M, Demchuk AM, Menon BK, Eesa M, Rempel JL, Thornton J, et al: Randomized assessment of rapid endovascular treatment of ischemic stroke. N Engl J Med 372:1019_ 1030,2015

8. Jovin TG, Chamorro A, Cobo E, de Miquel MA, Molina CA,
Rovira A, et al: Thrombectomy within 8 hours after symptom onset in ischemic stroke. N Engl J Med 372:2296-2306, 2015

9. Lee JS, Hong JM, Lee SJ, Joo IS, Lim YC, Kim SY: The combined use of mechanical thrombectomy devices is feasible for treating acute carotid terminus occlusion. Acta Neurochir (Wien) 155:635-641, 2013

10. Matsumoto H, Nishiyama H, Tetsuo Y, Takemoto H, Nakao $\mathrm{N}$ : Initial clinical experience using the two-stage aspiration technique (TSAT) with proximal flow arrest by a balloon guiding catheter for acute ischemic stroke of the anterior circulation. J Neurointerv Surg [epub ahead of print], 2016

11. Mokin M, Fargen KM, Primiani CT, Ren Z, Dumont TM, Brasiliense LB, et al: Vessel perforation during stent retriever thrombectomy for acute ischemic stroke: technical details and clinical outcomes. J Neurointerv Surg [epub ahead of print], 2016

12. Peschillo S, Diana F, Berge J, Missori P: A comparison of acute vascular damage caused by ADAPT versus a stent retriever device after thrombectomy in acute ischemic stroke: a histological and ultrastructural study in an animal model. J Neurointerv Surg [epub ahead of print], 2016

13. Saver JL, Goyal M, Bonafe A, Diener HC, Levy EI, Pereira VM, et al: Stent-retriever thrombectomy after intravenous t-PA vs. t-PA alone in stroke. N Engl J Med 372:2285-2295, 2015

14. Tsumoto T, Tsurusaki Y, Tokunaga S: Interaction between the stent strut and thrombus characterized by contrast-enhanced high-resolution cone beam CT during deployment of the Solitaire stent retriever. J Neurointerv Surg [epub ahead of print], 2016

15. Turk AS, Turner R, Spiotta A, Vargas J, Holmstedt C, Ozark $\mathrm{S}$, et al: Comparison of endovascular treatment approaches for acute ischemic stroke: cost effectiveness, technical success, and clinical outcomes. J Neurointerv Surg 7:666-670, 2015

\section{Disclosures}

The authors report no conflict of interest concerning the materials or methods used in this study or the findings specified in this paper.

\section{Author Contributions}

Conception and design: Lopes. Acquisition of data: Lopes. Analysis and interpretation of data: Okada. Drafting the article: Okada. Critically revising the article: Lopes, Crowley. Reviewed submitted version of manuscript: Matsuda, Chung, Crowley. Approved the final version of the manuscript on behalf of all authors: Lopes.

\section{Correspondence}

Demetrius K. Lopes, Department of Neurological Surgery, Rush University Medical Center, 1725 W Harrison St., Professional Bldg. Ste.855, Chicago, IL 60612.email: demetrius_lopes@ rush.edu. 